\title{
Mutation discovery in mice by whole exome sequencing
}

\author{
Heather Fairfield ${ }^{1}$, Griffith J Gilbert ${ }^{1}$, Mary Barter ${ }^{1}$, Rebecca R Corrigan², Michelle Curtain ${ }^{1}$, Yueming Ding ${ }^{3}$, \\ Mark D'Ascenzo ${ }^{4}$, Daniel J Gerhardt ${ }^{4}$, Chao He ${ }^{5}$, Wenhui Huang ${ }^{6}$, Todd Richmond ${ }^{4}$, Lucy Rowe ${ }^{1}$, Frank J Probst ${ }^{2}$, \\ David E Bergstrom', Stephen A Murray ${ }^{1}$, Carol Bult ${ }^{1}$, Joel Richardson', Benjamin T Kile, Ivo Gut ${ }^{8}$, Jorg Hager ${ }^{8}$, \\ Snaevar Sigurdsson ${ }^{9}$, Evan Mauceli ${ }^{9}$, Federica Di Palma ${ }^{9}$, Kerstin Lindblad-Toh ${ }^{9}$, Michael L Cunningham ${ }^{10}$, \\ Timothy C Cox ${ }^{10}$, Monica J Justice ${ }^{2}$, Mona S Spector ${ }^{5}$, Scott W Lowe ${ }^{5}$, Thomas Albert ${ }^{4}$, Leah Rae Donahue \\ Jeffrey Jeddeloh ${ }^{4}$, Jay Shendure ${ }^{10}$ and Laura G Reinholdt ${ }^{*}$
}

\begin{abstract}
We report the development and optimization of reagents for in-solution, hybridization-based capture of the mouse exome. By validating this approach in a multiple inbred strains and in novel mutant strains, we show that whole exome sequencing is a robust approach for discovery of putative mutations, irrespective of strain background. We found strong candidate mutations for the majority of mutant exomes sequenced, including new models of orofacial clefting, urogenital dysmorphology, kyphosis and autoimmune hepatitis.
\end{abstract}

\section{Background}

Phenotype-driven approaches in model organisms, including spontaneous mutation discovery, standard $\mathrm{N}$-ethyl- $\mathrm{N}$ nitrosourea (ENU) mutagenesis screens, sensitized screens and modifier screens, are established approaches in functional genomics for the discovery of novel genes and/or novel gene functions. As over $90 \%$ of mouse genes have an ortholog in the human genome [1], the identification of causative mutations in mice with clinical phenotypes can directly lead to the discovery of human disease genes. However, mouse mutants with clinically relevant phenotypes are not maximally useful as disease models until the underlying causative mutation is identified. Until recently, the gene discovery process in mice has been straightforward, but greatly hindered by the time and expense incurred by high-resolution recombination mapping. Now, the widespread availability of massively parallel sequencing [2] has brought about a paradigm shift in forward genetics by closing the gap between phenotype and genotype.

Both selective sequencing and whole genome sequencing are robust methods for mutation discovery in the mouse genome [3-5]. Nonetheless, the sequencing and analysis of whole mammalian genomes remains computationally

\footnotetext{
* Correspondence: laura.reinholdt@jax.org

'The Jackson Laboratory, 600 Main St, Bar Harbor, ME 04609, USA Full list of author information is available at the end of the article
}

burdensome and expensive for many laboratories. Targeted sequencing approaches are less expensive and the data are accordingly more manageable, but this technique requires substantial genetic mapping and the design and purchase of custom capture tools (that is, arrays or probe pools) [4]. Targeted sequencing of the coding portion of the genome, the 'exome', provides an opportunity to sequence mouse mutants with minimal mapping data and alleviates the need for a custom array/probe pool for each mutant. This approach, proven to be highly effective for the discovery of coding mutations underlying single gene disorders in humans [6-12], is particularly relevant to large mutant collections, where high-throughput gene discovery methods are desirable.

Currently, there are nearly 5,000 spontaneous and induced mouse mutant alleles with clinically relevant phenotypes catalogued in the Mouse Genome Informatics database [13]. The molecular basis of the lesions underlying two-thirds of these phenotypes is currently unknown. For the remaining one-third that have been characterized, the Mouse Genome Informatics database indicates that $92 \%$ occur in coding sequence or are within 20 bp of intron/exon boundaries, regions that are purposefully covered by exome targeted re-sequencing. While this estimate is impacted by an unknown degree of ascertainment bias (since coding or splice site mutations are easier to find 
and hence reported and since many uncharacterized mutations remain so because they are understudied), we anticipated that exome sequencing would still be likely to capture a considerable percentage of spontaneous and induced mouse mutations. Therefore, to significantly reduce the time, effort, and cost of forward genetic screens, we developed a sequence capture probe pool representing the mouse exome. Here, we describe the utility of this tool for exome sequencing in both wild-type inbred and mutant strain backgrounds, and demonstrate success in discovering both spontaneous and induced mutations.

\section{Results and discussion}

\section{Mouse exome content and capture probe design}

The coding sequence selected for the mouse exome probe pool design includes 203,225 exonic regions, including microRNAs, and collectively comprises over $54.3 \mathrm{Mb}$ of target sequence (C57BL/6J, NCBI37/mm9). The design was based on a unified, Mouse Genome Database-curated gene set, consisting of non-redundant gene predictions from the National Center for Biotechnology Information (NCBI), Ensembl and The Vertebrate Genome Annotation (VEGA) database [13]. The gene list is available at [14]. To manage the size of the probe pool and to avoid non-uniquely mappable regions, we excluded olfactory receptors and pseudogenes from the target sequence. In cases where an exon contained both UTR and coding sequence, the UTR sequence was included in the design. Two DNA probe pools, alpha and beta prototypes, were ultimately designed and tested. To maximize the uniformity of the sequencing libraries after capture, re-sequencing data from the alpha prototype design were empirically studied and used to inform a coverage re-balancing algorithm. That algorithm altered the probe coverage target ratio of a second design (beta prototype) in an attempt to decrease over-represented sequence coverage, and increase under-represented sequence coverage. The target (primary design) coordinates and the coordinates of the capture probes in the beta design are available at [15]. The summary statistics for each probe pool are shown in Additional file 1.

\section{Exome capture performance and optimization}

To test the alpha and beta exome probe pools and to determine whether strain background adversely influenced performance, exomes from four commonly used inbred strains (C57BL/6J, 129S1/SvImJ, BALB/cJ and $\mathrm{C} 3 \mathrm{H} / \mathrm{HeJ}$ ) were captured and re-sequenced (Table 1). Overall, capture sensitivity was high, with just one lane of $2 \times 40$-bp paired-end sequencing $(2 \times 40 \mathrm{bp} \mathrm{PE})$ resulting in $>96 \%$ of the targeted bases covered. The capture specificity was also high with $>75 \%$ reads mapping to targeted bases. Importantly, the sequencing data were significantly enriched, not only for coding sequence but also for flanking splice acceptor and donor sites, where deleterious mutations are frequently found (Figure 1). Genetic background only modestly impacted the sensitivity and specificity of the capture probe pools. The variation between strains was greater than within a strain (Table 1); however, the scale of the inter-strain differences observed suggests that a pool based upon exclusively the $\mathrm{mm} 9$ reference would be functional with any Mus musculus background.

The beta design was made using a proprietary rebalancing algorithm from Roche NimbleGen (Madison, WI, USA) that removes probes from targets with high coverage and adds probes to low coverage targets in order to maximize coverage across targets. In addition to testing the beta design by exome capture and $2 \times 40$ bp PE Illumina sequencing of four different inbred strains, the beta design was also tested with four independent captures of C57BL/6J female DNA and sequenced on the Illumina GAII platform, $2 \times 76$ bp PE. The most dramatic improvement was observed in the fraction of targeted bases covered at $20 \times$ or more where the increase in uniformity resulted in $12 \%$ improvement (Additional file 2).

\section{Sequencing of mutant exomes}

To determine the efficacy of the probe pools for mutant exome re-sequencing and mutation discovery, 15 novel mouse mutant exomes and 3 controls were captured and sequenced at multiple sites using different Illumina platforms (Illumina GAIIx, Illumina HiSeq, and both $2 \times 76$ bp and $2 \times 100$-bp PE libraries). The mutants were selected based on several parameters, including research area, mode of inheritance (dominant and recessive), strain background, and mutation type (induced and spontaneous). Where appropriate, homozygous samples were captured and sequenced (Additional file 3). In all cases, the beta exome pools provided improved capture uniformity. In the majority of cases, $>97 \%$ of targeted bases were covered by at least one read $(1 \times)$. Approximately 45 million 100-bp PE reads were sufficient, on average, to provide at least 5 reads coverage of $95 \%$ of target bases (Table 2; Additional file 4), which is sufficient for detection of recessive mutations in homozygous samples. To confidently call heterozygous alleles, at least $15 \times$ coverage is preferable [4], and these data show that more than 58 million, 100-bp PE reads are likely required to obtain a minimum of 15 reads across $95 \%$ of target bases. Therefore, we anticipate that sample indexing schemes may soon enable as many as four exomes to be multiplexed per lane of an Illumina HiSeq run using the most current reagents. The raw sequencing data for mutant and inbred strains are available from the NCBI Sequence Read Archive (accession number [SRP007328]). 
Table 1 Direct comparison of coverage statistics from exome re-sequencing $(2 \times 40 \mathrm{bp}$, Illumina) of four inbred strains with two exome probe pool designs, alpha and beta

\begin{tabular}{lcccccccc}
\hline & \multicolumn{7}{c}{ Sample } \\
\cline { 2 - 8 } & C57BL/6J & C57BL/6J & 129S1/SvlmJ & 129S1/SvlmJ & BALB/cJ & BALB/cJ & C3H/HeJ & C3H/HeJ \\
\hline Exome version & Alpha & Beta & Alpha & Beta & Alpha & Beta & Alpha & Beta \\
Quantitative PCR & 161.81 & 168.53 & 129.43 & 95.75 & 168.92 & 165.08 & 168.38 & 92.00 \\
Target exons & 203,225 & 203,224 & 203,225 & 203,224 & 203,225 & 203,224 & 203,225 & 203,224 \\
Target bases & $54,367,346$ & $54,367,244$ & $54,367,346$ & $54,367,244$ & $54,367,346$ & $54,367,244$ & $54,367,346$ & $54,367,244$ \\
Target bases covered & $52,266,238$ & $53,273,874$ & $51,746,839$ & $52,508,881$ & $51,828,334$ & $52,862,662$ & $52,136,965$ & $51,460,949$ \\
Percentage target bases covered & 96.14 & 97.99 & 95.18 & 96.58 & 95.33 & 97.23 & 95.90 & 94.65 \\
Target bases not covered & $2,101,108$ & $1,093,370$ & $2,620,507$ & $1,858,363$ & $2,539,012$ & $1,504,582$ & $2,230,381$ & $2,906,295$ \\
Percentage target bases not covered & 3.86 & 2.01 & 4.82 & 3.42 & 4.67 & 2.77 & 4.10 & 5.35 \\
Median coverage & 18.45 & 20.74 & 17.93 & 16.37 & 18.05 & 20.75 & 18.76 & 7.86 \\
Total reads & $60,582,097$ & $60,207,746$ & $64,258,556$ & $44,434,168$ & $64,495,816$ & $63,740,186$ & $64,959,026$ & $25,760,946$ \\
NC80 & 0.28 & 0.37 & 0.25 & 0.33 & 0.25 & 0.31 & 0.29 & 0.32 \\
1/NC80 & 3.53 & 2.71 & 4.03 & 3.02 & 3.96 & 3.27 & 3.50 & 3.13 \\
\hline
\end{tabular}

$1 / \mathrm{NC} 80$ is the fold 80 penalty, which represents the fold of over-sequencing necessary to move $80 \%$ of the below median bases to median.

\section{Mapping and variant calling}

Mapping to the mouse reference sequence (C57BL/6J, $\mathrm{NCBI} 37 / \mathrm{mm} 9$ ) and subsequent variant calling resulted in a number of single nucleotide variants (SNVs) and insertions/deletions (INDELs) ranging from approximately 8,000 (C57BL/6J background) to over 200,000 (for more divergent strain backgrounds) variant calls per mutant exome, depending on strain background and depth of coverage. Generally, approximately two-thirds of the variants called were SNVs, rather than INDELS. However, in mutants on the C57BL/6J background, this ratio was closer to approximately one-half (Additional file 3 ). This is not surprising given that a large proportion of false positive calls from reference guided assembly are INDELs and the number of true variants in any $\mathrm{C} 57 \mathrm{BL} / 6 \mathrm{~J}$ exome is expected to be low because the mouse reference strain is, primarily, C57BL/6J. The one exception was mutant 12860 (nert), which was reported to be on a C57BL/6J background; however, the relatively large number of variants detected in this mutant exome could indicate that the reported strain background is likely incorrect.

\section{Variant annotation and nomination of candidate mutations}

The variant data were fully annotated according to genomic position, SNV quality, allele ratio (number of reads containing variant allele/number of reads containing reference allele), and overlap with current genome

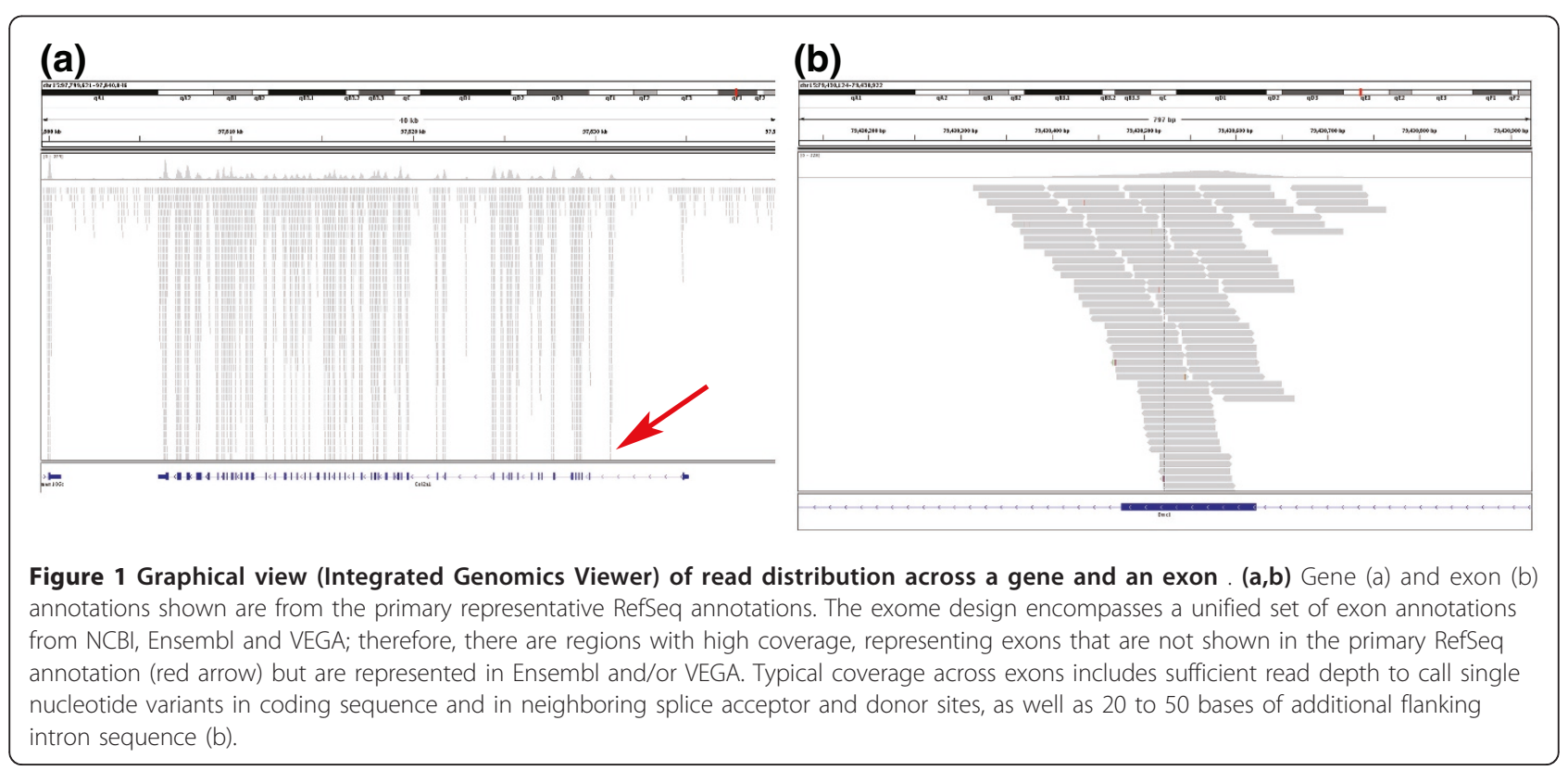


Table 2 Representative coverage statistics from exome re-sequencing $(2 \times 100 \mathrm{bp})$ of six mutant strains

\begin{tabular}{|c|c|c|c|c|c|c|}
\hline & \multicolumn{6}{|c|}{ Sample } \\
\hline & 5330 (hbck) & 6246 (sunk) & 8568 (lear) & 12856 (shep) & $13782($ aphl) & 13716 (vgim) \\
\hline Targeted exons & 203,224 & 203,224 & 203,224 & 203,224 & 203,224 & 203,224 \\
\hline Final target bases & $54,367,244$ & $54,367,244$ & $54,367,244$ & $54,367,244$ & $54,367,244$ & $54,367,244$ \\
\hline Target bases covered & $52,934,978$ & $52,493,811$ & $52,832,014$ & $52,647,881$ & $52,664,921$ & $53,004,900$ \\
\hline Percentage target bases covered & 97.37 & 96.55 & 97.18 & 96.84 & 96.87 & 97.49 \\
\hline Target bases not covered & $1,432,266$ & $1,873,433$ & $1,535,230$ & $1,719,363$ & $1,702,323$ & $1,362,344$ \\
\hline Percentage target bases not covered & 2.63 & 3.45 & 2.82 & 3.16 & 3.13 & 2.51 \\
\hline Total reads $^{\mathrm{a}}$ & $39,675,108$ & $39,641,830$ & $31,817,686$ & $42,405,386$ & $59,956,764$ & $67,359,382$ \\
\hline Number of reads in target regions & $23,319,015$ & $23,335,916$ & $19,211,748$ & $25,227,205$ & $36,227,876$ & $39,948,582$ \\
\hline Percentage reads in target regions & 58.77 & 58.87 & 60.38 & 59.49 & 60.42 & 59.31 \\
\hline Average coverage & 32.72 & 32.59 & 26.75 & 35.32 & 50.78 & 56.31 \\
\hline Median coverage & 30.33 & 30.02 & 23.23 & 33.02 & 46.61 & 50.02 \\
\hline Coverage at $20 x$ & 76.4 & 73.6 & 61.9 & 77.5 & 85.8 & 88 \\
\hline Coverage at $10 x$ & 92.1 & 89.3 & 87.1 & 90.7 & 92.9 & 94.5 \\
\hline Coverage at $5 x$ & 95.7 & 93.8 & 94.3 & 94.4 & 95.1 & 96.2 \\
\hline Coverage at $1 \times$ & 97.4 & 96.6 & 97.2 & 96.8 & 96.9 & 97.5 \\
\hline NC80 & 0.51 & 0.47 & 0.46 & 0.49 & 0.47 & 0.46 \\
\hline $1 / N C 80$ & 1.94 & 2.13 & 2.18 & 2.06 & 2.13 & 2.17 \\
\hline
\end{tabular}

$1 / \mathrm{NC} 80$ is the fold 80 penalty, which represents the fold of over sequencing necessary to move $80 \%$ of the below median bases to median. Coverage statistics for all samples sequenced can be found in Additional file $3 .{ }^{\mathrm{a}} 2 \times 100 \mathrm{bp}$, Illumina HiSeq.

annotations, including NCBI Reference Sequence (RefSeq)/Ensembl genes, exons, introns, splice sites, and known SNVs, INDELs (the Single Nucleotide Polymorphism database, dbSNP). In each case, existing linkage data were used to determine map positions and the analysis was then limited to those regions. The existing linkage data ranged from coarse (chromosomal linkage) to fine (regions of $<10$ to $20 \mathrm{Mb}$ ) (Additional file 3). The most likely causative mutations for each mutant sample and for a control C57BL/6J exome were nominated using the annotations as shown in Table 3. Specifically, novel (when compared to dbSNP) protein coding or splice site variants falling within mapped regions, with expected allele ratios ( $>0.95$ for homozygous variants and $>0.2$ for heterozygous variants) were given priority for validation by re-sequencing of additional mutant and unaffected samples. To further reduce the validation burden, we found that comparison of unrelated exome sequencing data sets and comparison to the Sanger Institute Mouse Genomes data [16] allowed for significant reduction in validation burden, as any variants common between these data sets represent common variants that are shared between related strains or systematic false positives arising from mapping the data back to the reference sequence. Similar to what has been observed in human exome sequencing, the latter can be caused by repetitive or closely related sequences (paralogs) or underlying deficiencies in the reference sequence. For comparison, the alignment data from the C57BL/6J beta exome shown in Table 1 were subjected to variant calling and annotation. Interestingly, 17 variants passed filters in a C57BL/6J exome (Table 3), expected to be most similar to the reference genome, which is also primarily C57BL/6J. Comparison of these variants with the high throughput sequencing data for 17 inbred strains available from Sanger Mouse Genomes Project revealed three exonic SNVs unique to the C57BL/6J exome. We predict that the remaining 14 variants calls are false positive calls due to mapping errors, which can arise in regions where there is underlying deficiency in the reference sequence or in regions that share sequence similarity (that is, paralogs). These regions are apparent when viewing alignments as regions that contain a preponderance of non-uniquely mapped reads, gaps, or regions that contain apparent heterozygosity in samples that are known to be homozygous (as is the case with the inbred strain data from the Sanger Mouse Genomes project, where each strain was subjected to at least 200 generations of brother $\times$ sister intercrossing prior to sequencing; Additional file 5).

\section{Validation of putative causative mutations}

Using this approach, only one or two variants were nominated for validation in each of nine mutant exomes. Four of these mutants represented ENU-generated lines, while five were spontaneous mutants. In a few cases, the single variant nominated for validation proved to be the likely causative mutation. For example, the single SNV nominated for validation in the bloodline mutant correlated with the phenotype when additional affected and 
Table 3 Analysis of annotated variant data from mutant exome sequencing

\begin{tabular}{|c|c|c|c|c|c|c|c|c|c|c|}
\hline $\begin{array}{l}\text { Mutant } \\
\text { number } \\
\text { (allele) }\end{array}$ & $\begin{array}{l}\text { Inheritance/ } \\
\text { phenotype }\end{array}$ & $\begin{array}{l}\text { Mutation } \\
\text { type: strain } \\
\text { background }\end{array}$ & $\begin{array}{c}\text { Variants } \\
\text { called }\end{array}$ & $\begin{array}{c}\text { In gene } \\
\text { (introns, } \\
\text { exons) }\end{array}$ & $\begin{array}{l}\text { Novel } \\
\text { SNVs }^{\text {a }}\end{array}$ & $\begin{array}{c}\text { Overlap } \\
\text { with map } \\
\text { position }\end{array}$ & $\begin{array}{l}\text { Allele } \\
\text { ratio }^{\text {b }}\end{array}$ & $\begin{array}{l}\text { Non-synonymous } \\
\text { coding variants, } \\
\text { splice sites }\end{array}$ & Unique $^{c}$ & $\begin{array}{l}\text { Putative } \\
\text { mutation }\end{array}$ \\
\hline $\begin{array}{l}12874 \\
\text { (bloodline) }\end{array}$ & $\begin{array}{l}\text { Recessive/ } \\
\text { metabolic }\end{array}$ & $\begin{array}{l}\text { Spontaneous: } \\
\text { stock (mixed } \\
\text { B6) }\end{array}$ & 134,205 & 116,120 & 35,469 & 350 & 155 & 29 & 1 & Map3k11, E293K \\
\hline $\begin{array}{l}12724 \\
\text { (Cleft) }\end{array}$ & $\begin{array}{l}\text { Dominant/ } \\
\text { craniofacial }\end{array}$ & $\begin{array}{l}\text { ENU: C57BL/6J, } \\
\text { C3HeB/FeJ }\end{array}$ & 49,367 & 36,037 & 10,873 & 83 & 53 & 19 & 2 & Col2a1, Q713Stop \\
\hline repro7 & $\begin{array}{l}\text { Recessive/ } \\
\text { reproductive }\end{array}$ & $\begin{array}{l}\text { ENU: C57BL/6J, } \\
\text { C3H/HeJ, Cast/ } \\
\text { EiJ }\end{array}$ & 410,333 & 185,999 & 87,568 & 799 & 47 & 7 & 1 & Prdm9, Q478Stop \\
\hline $\begin{array}{l}5330 \\
(h p b k)\end{array}$ & $\begin{array}{l}\text { Recessive/ } \\
\text { skeletal }\end{array}$ & ENU: C57BL/6J & 8,516 & 6,167 & 4,589 & 35 & 3 & 2 & 2 & $\begin{array}{l}\text { Notch3, splice } \\
\text { donor site (G to } \\
\text { A), intron } 31\end{array}$ \\
\hline $\begin{array}{l}13716 \\
\text { (vgim) }\end{array}$ & $\begin{array}{l}\text { Recessive/ } \\
\text { reproductive }\end{array}$ & $\begin{array}{l}\text { Spontaneous: } \\
\text { C57BL/6J }\end{array}$ & 10,134 & 7,346 & 5,533 & 117 & 6 & 3 & 2 & Lhfp/2, G102E \\
\hline 8568 (lear) & $\begin{array}{l}\text { Recessive/ } \\
\text { small ears }\end{array}$ & $\begin{array}{l}\text { Spontaneous: } \\
\text { C57BL/6J }\end{array}$ & 8,219 & 5,715 & 1,889 & 12 & 1 & 1 & 1 & $\begin{array}{l}\text { Prkra, intron 5, } \\
\text { splice donor }\end{array}$ \\
\hline $\begin{array}{l}12856 \\
\text { (shep) }\end{array}$ & $\begin{array}{l}\text { Recessive/ } \\
\text { metabolic }\end{array}$ & $\begin{array}{l}\text { Spontaneous: } \\
\text { A/J }\end{array}$ & 164,116 & 59,067 & 16,930 & 454 & 177 & 83 & 1 & Relb, Q334K \\
\hline 111Jus74 & Recessive & ENU: B6, 129 & 230,896 & 52,628 & 14,448 & 344 & 37 & 4 & 2 & $\begin{array}{l}\text { Rundc3a, Y46F; } \\
\text { Nek8, V343E }\end{array}$ \\
\hline $\begin{array}{l}4235 \\
\text { (Sofa) }\end{array}$ & $\begin{array}{l}\text { Dominant, } \\
\text { craniofacial }\end{array}$ & $\begin{array}{l}\text { Spontaneous: } \\
\text { C57BL/6J, AKR/ } \\
\text { J }\end{array}$ & 134,207 & 116,122 & 35,471 & 346 & 310 & 121 & 1 & $\begin{array}{l}\text { Pfas, } \\
\text { H1194_G1198del }\end{array}$ \\
\hline C57BL/6J & NA & None & 5,980 & 3,953 & 3,132 & NA & 538 & 17 & 3 & NA \\
\hline $\begin{array}{l}13716 \\
\text { (vgim) }\end{array}$ & $\begin{array}{l}\text { Recessive/ } \\
\text { reproductive }\end{array}$ & $\begin{array}{l}\text { Spontaneous: } \\
\text { C57BL/6J }\end{array}$ & 10,134 & 7,346 & 5,533 & NA & 940 & 97 & 38 & NA \\
\hline
\end{tabular}

${ }^{a}$ Compared to dbSNP. ${ }^{b}>0.95$ for homozygous samples, $>0.2$ for heterozygous samples. ${ }^{c}$ compared to unrelated exome data sets. NA, not available.

unaffected samples were tested (Figure 2a). The SNV is a missense mutation causing an amino acid change (E293K) in Map3K11, a gene that encodes a mitogen-activated protein kinase kinase kinase that is involved in a variety of cellular signaling cascades. Importantly, mice homozygous for a targeted null mutation in Map3k11 have the characteristic epidermal midline defect that is also observed in bloodline homozygotes [17], further implicating the missense mutation found as the causative mutation. Unlike bloodline homozygotes, Map3K11-/- mice are viable and tooth pulp necrosis has not been reported [17], indicating that the spontaneous mutation may be sensitive to strain background effects. However, further work is needed to establish the underlying mechanisms influencing these phenotypic differences.

In some cases, more than one potentially damaging variant was found to correlate with the phenotype when additional affected and unaffected animals from the pedigree were genotyped (Table 3 ). In two cases, $h p b k$ and vim, where more than one variant was found, only one variant could be validated while the other variants were false positives. In two cases where more than one potentially damaging variant was found, both were validated. Not surprisingly, these cases were ENU-induced mutant exomes (Cleft and l11Jus74) and ENU is known to cause mutations at a rate of greater than 1 in 750 per locus per gamete [18] at doses of $85 \mathrm{mg} / \mathrm{kg}$. Cleft is a dominant craniofacial ENU mutation that causes cleft palate. Of the two variants that were nominated for validation, both were SNVs residing in Col2a1, a gene coding for type II procollagen. Both SNVs reside within $10 \mathrm{~kb}$ of each other (Chr15:97815207 and Chr15:97825743) in Col2a1, a gene coding for type II procollagen, and not surprisingly were found to be concordant with the phenotype when multiple animals from the pedigree were genotyped. The most likely causative lesion ( $\mathrm{G}$ to A at Chr15:97815207) is a nonsense mutation that introduces a premature stop codon at amino acid 645. The second closely linked variant is an $\mathrm{A}$ to $\mathrm{T}$ transversion in intron 12 that could potentially act as a cryptic splice site. However, since RTPCR did not reveal splicing abnormalities, it is more likely that the nonsense mutation is the causative lesion (Figure 2b). Mice homozygous for targeted deletions in Col2a1 and mice homozygous for a previously characterized, spontaneous mis-sense mutation, Col2a1 $1^{\text {sedc }}$, share similar defects in cartilage development to Cleft mutants, including recessive peri-natal lethality and orofacial clefting $[19,20]$, providing further support that the Cleft phenotype is the result of a mutation in Col2a1.

The l11Jus 74 mutation was isolated in a screen for recessive lethal alleles on mouse chromosome 11 using a 129.Inv(11)8Brd ${ }^{\text {Trp53-Wnt3 }}$ balancer chromosome $[21,22]$. 

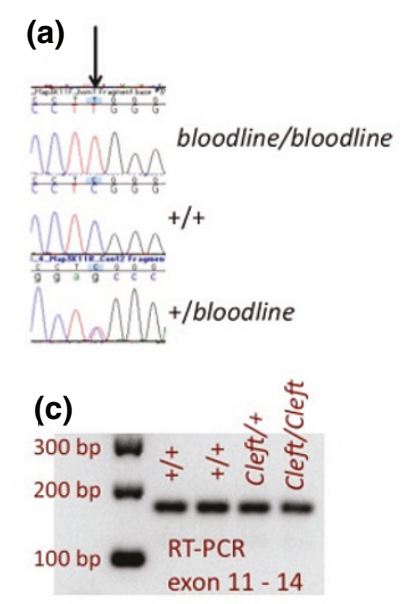

(b)
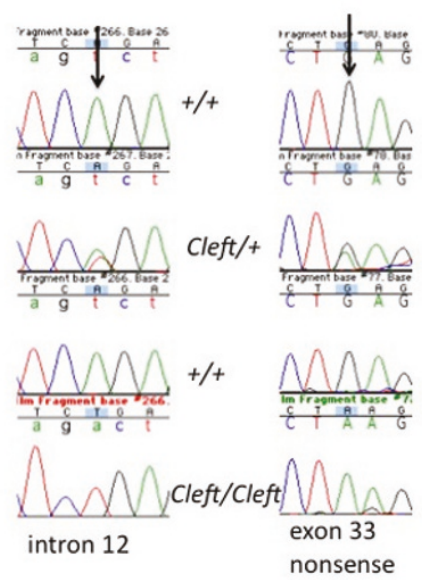

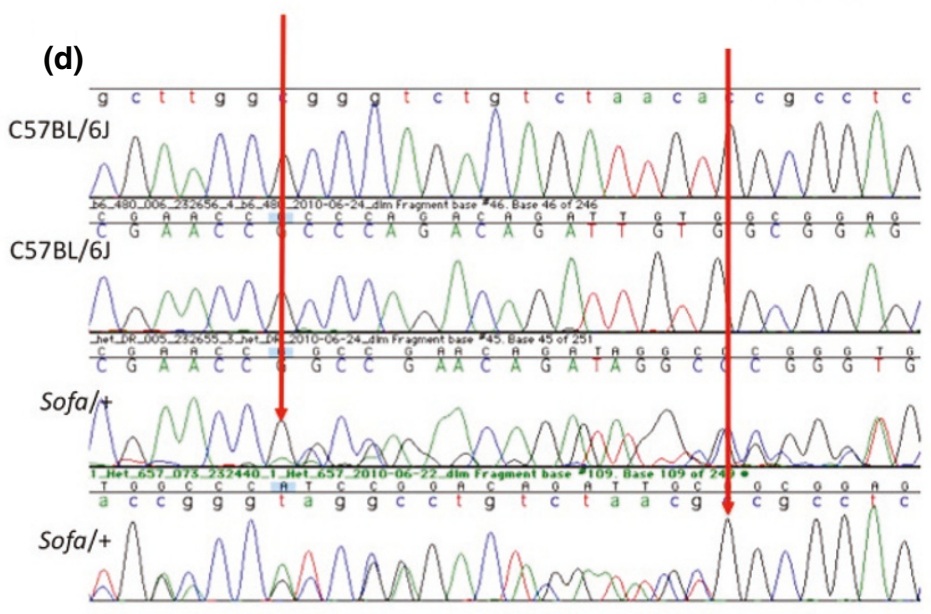

(e)

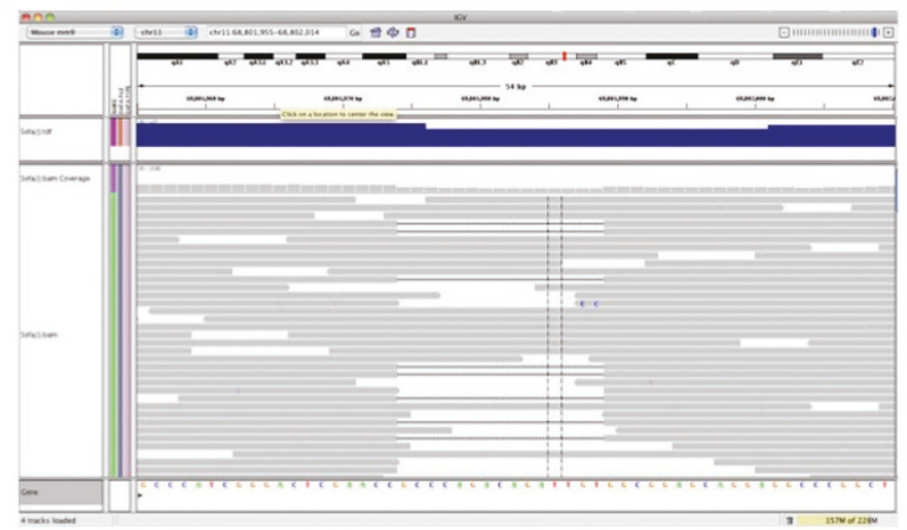

Figure 2 Examples of validated mutations discovered in mutant exome data. The bloodline mutation is a recessive mutation that causes a distinctive dorsal epidermal defect and tooth pulp necrosis. Exome sequencing revealed a G to A mutation in Map3K11 (mitogen-activated protein kinase kinase kinase 11). (a) PCR and sequencing of additional mutant (bloodline/bloodline) and unaffected (+/+ or $+/-)$ animals provided additional support for this putative mutation. The 'Cleft' mutation is an ENU mutation that arose on C57BL/6J. The mutation causes a dominant craniofacial phenotype and recessive perinatal lethality with characteristic cleft palate. (b) Sanger sequencing confirmed the presence of two closely linked mutations in multiple cleft/+ and cleft/cleft samples and the absence of these mutations in +/+ littermate samples. (c) Of the two mutations found, the intron mutation has the potential to cause splicing defects, although it is less likely to contribute to the phenotype since RT-PCR shows no indication of defective splicing mutant samples. The 'Sofa' mutation is a spontaneous mutation that arose on C57BL/6J, causing a dominant craniofacial phenotype and recessive perinatal lethality. (d) Sanger sequencing of heterozygous and control samples confirmed the presence of a 15-bp deletion in Pfas, FGAR amidotransferase. (e) Reads from the mutant, deletion-bearing allele successfully mapped to Pfas using BWA (Burrows-Wheeler aligment tool) and the deletion was called using SAMtools [25] with an allele ratio of 0.2 . 
The screen was performed as described previously using C57BL/6J ENU-treated males, mated to the balancer, which was generated in 129S5SvEv embryonic stem cells. Embryos from the 111 Jus 74 line were analyzed from timed matings, as previously described [23], to determine that homozygotes die perinatally. Two potentially causative missense mutations were found in Nek8 (NIMA (never in mitosis gene a)-related expressed kinase 8; V343E) and Rundc3a (Run domain containing 3a; Y46F). Mutations in Nek8 cause polycystic kidney disease, but no phenotypes have been ascribed to mutations in Rundc3a. Although the cause of death of l11Jus74 homozygotes has not been determined, polycystic kidneys have not been observed, making the most likely lesion to result in perinatal death Rundc3a, although the Nek8 mutation may cause a delayed onset phenotype.

For all four of the ENU-induced mutant exomes sequenced, putative causative mutations were nominated and validated. Mutations induced by ENU are usually single nucleotide substitutions. The high sensitivity of current analytical pipelines for detecting single nucleotide substitutions (and particularly homozygous substitutions), combined with the propensity of damaging single nucleotide substitutions to occur in coding sequences, likely explains the high success rate of exome sequencing for detecting induced lesions. Similarly, Boles et al. [24] showed that targeted sequencing of exons and highly conserved sequences from ENU mutants mapping to chromosome 11 yielded a high success rate, with candidate mutations nominated in nearly $75 \%$ of mutants.

While mutations induced by mutagens like ENU are known to cause single nucleotide substitutions, spontaneous mutations are the result of a variety of lesions, including single nucleotide substitutions, small INDELS and larger deletions or insertions of mobile DNA elements. Of the nine potentially damaging coding or splicing mutations discovered in this set of mutant exomes, the spontaneous Sofa mutant was the only one for which a single nucleotide substitution was not discovered. Instead, a 15-bp deletion in Pfas (Table 3; Figure 2d,e) was found, demonstrating that small deletions in coding sequence can be discovered using this approach.

Interestingly, the allele ratio for the Sofa deletion was 0.2 , which is lower than expected for a heterozygote; therefore, a stringent cutoff of 0.5 or even 0.35 , which we previously found was sufficient for calling heterozygous variants at approximately $80 \%$ confidence [4], would have eliminated this variant from consideration. The lower allele ratio is likely the result of bias in either the capture of the INDEL-containing fragments, and/or the ability to appropriately map some of the INDEL-bearing reads. Since the library fragments are larger than both the probes and the exons they target and because each target is tiled with multiple probes, there are expected to be perfect match probes somewhere within an exon for nearly every allele despite the presence of an INDEL. Consequently, we favor a mapping problem as the major driver for the lower than expected allele ratio observed (Figure 2e). Longer reads may alleviate some systematic issues associated with discovering relevant deletions or insertions. A 15-bp deletion would maximally comprise a mismatch of nearly $38 \%$ along a 40 -bp read, but only $20 \%$ within a 76 -bp read. Large gaps $(20 \%$ or more of the read) would impose a stiff mapping penalty on that end of read pairs. Presumably, longer reads (100 bp or longer) would incur lower penalties, thereby moderating adverse mapping effects.

Approximately $10 \%$ of known deleterious mutations in the mouse genome affect the conserved splice acceptor or donor sites (Table 4), which include the two intronic nucleotides immediately flanking each exon. Of the putative mutations discovered in this set of 15 mutant exomes, three candidates were found in or immediately adjacent to the conserved splice acceptor or donor sites (Cleft, lear, and hpbk), demonstrating that exome sequencing provides sufficient coverage of flanking intron sequence to positively identify potentially damaging, noncoding mutations in the intron sequences immediately flanking target exons.

\section{Traditional genetic mapping and exome sequencing}

In all cases, either coarse mapping data (chromosomal linkage) or a fine map position $(<20 \mathrm{Mb})$ was available to guide analysis and ease validation burden (Additional file 3 ). For example, the shep mutation was previously linked to chromosome 7 (approximately $152 \mathrm{Mb}$ ), while repro7 was fine mapped to a $4.5 \mathrm{Mb}$ region on chromosome 17. The mapping of shep to chromosome 7 was accomplished using a group of 20 affected animals, while the fine mapping of repro7 to a $4.5 \mathrm{Mb}$ region on chromosome 17 required the generation of 524 F2 animals, requiring over a year of breeding in limited vivarium space. In both cases, the mapping data coupled with the additional filtering of annotated data, as shown in Table 3 , significantly reduced the validation burden to a single variant. Therefore, highthroughput sequencing (exome or whole genome) represents a cost efficient alternative to fine mapping by recombination, especially in cases where vivarium space and time are limited resources.

In the absence of chromosomal linkage, the validation burden is significantly larger. For example, the vgim mutant exome was reanalyzed without utilizing mapping information (Table 3, last row) and 38 variants were nominated for validation. Addition of just the chromosomal linkage data for vgim (chromosome 13), but not the fine mapping data (chr13:85473357-96594659) reduces the validation burden to two candidates. Therefore, coarse mapping to establish chromosomal linkage 
Table 4 In silico analysis of all induced or spontaneous alleles $(4,984)$ with phenotypes reported in the Mouse Genomes Database [1]

\begin{tabular}{|c|c|}
\hline Mutation & $\begin{array}{l}\text { Number of } \\
\text { alleles }\end{array}$ \\
\hline Unknown or uncharacterized & 3,105 \\
\hline $\begin{array}{l}\text { Introns, UTRs, regulatory regions (including instances where the lesion is not known but coding sequence has been sequenced), } \\
\text { cryptic splice sites, inversions }\end{array}$ & 150 \\
\hline Exons (single nucleotide substitutions, deletions, insertions) & 1,581 \\
\hline Conserved splice acceptor or donor & 148 \\
\hline
\end{tabular}

This analysis shows that the vast majority of induced or spontaneous alleles that have been characterized at the molecular level (1,879) are mutations in coding sequence or conserved splice acceptor/splice donor sites.

provides significant reduction in validation burden at minimal additional animal husbandry cost and time. In the absence of mapping data and/or when mutations arise on unusual genetic backgrounds, exome sequencing of additional samples (affected animal and parents) would similarly reduce the validation burden to just one or a few variants.

\section{Limitations of exome sequencing for mutation discovery}

Using this technology, we validated putative causative coding mutations in 9 of the 15 mutant exomes examined. For the remaining six mutants, candidate mutations were found in UTRs or were not found at all (Table 5). For Alf, nert and aphl, candidate mutations were found in UTRs, and interestingly, in nearly every case, these candidate mutations are in genes not currently associated with any mouse phenotype. For the other three mutants, frg, stn and sunk, no candidate mutations were found in protein coding sequence, splice sites or in UTRs. Failure to identify the candidate causative mutations most likely indicates that these mutations reside in non-coding, regulatory regions or unannotated coding sequence that is not included in the current exome capture design. An additional possibility is that the underlying mutations do reside in the targeted regions, but are simply not revealed using standard mapping and SNP calling, which is clearly biased towards the discovery of single nucleotide substitutions and small INDELs. Robust computational methods for finding larger insertions and deletions and/or translocations via high-throughput sequencing data are not widely available and the absence of these tools limits spontaneous mutation discovery by any means, whether exome or whole genome sequencing.

In a parallel effort, we used targeted sequencing of contiguous regions to discover spontaneous mutations that have been mapped to regions of $10 \mathrm{Mb}$ or less. Interestingly, the success rate for nominating putative mutations via targeted sequencing of contiguous regions was comparable to that of exome sequencing (at approximately $60 \%)$, demonstrating that despite the availability of

Table 5 Validation of putative causative coding mutations in 15 mutant exomes

\begin{tabular}{|c|c|c|c|c|c|c|c|c|c|c|c|}
\hline $\begin{array}{l}\text { Mutant } \\
\text { number } \\
\text { (allele) }\end{array}$ & $\begin{array}{l}\text { Inheritance/ } \\
\text { phenotype }\end{array}$ & $\begin{array}{l}\text { Strain } \\
\text { background }\end{array}$ & $\begin{array}{l}\text { Variants } \\
\text { called }\end{array}$ & $\begin{array}{l}\text { In gene } \\
\text { (introns, } \\
\text { exons) }\end{array}$ & $\begin{array}{l}\text { Novel } \\
\text { SNVs }^{a}\end{array}$ & $\begin{array}{l}\text { Overlap } \\
\text { with } \\
\text { map } \\
\text { position }\end{array}$ & $\begin{array}{l}\text { Allele } \\
\text { ratio }^{b}\end{array}$ & $\begin{array}{c}\text { Non- } \\
\text { synonymous } \\
\text { coding } \\
\text { variants, } \\
\text { splice sites }\end{array}$ & Unique $^{c}$ & $\begin{array}{l}\text { Validation } \\
\text { of coding/ } \\
\text { splice } \\
\text { variants }\end{array}$ & $\begin{array}{l}\text { Variants in } \\
\text { UTRs }\end{array}$ \\
\hline $\begin{array}{l}5413 \\
(P / p s)\end{array}$ & $\begin{array}{l}\text { Dominant/ } \\
\text { craniofacial }\end{array}$ & $\begin{array}{l}\text { Spontaneous: } \\
\text { C57BL/6J, } \\
\text { 129S1/SvImJ }\end{array}$ & 13,453 & 3,271 & 1,821 & 200 & 129 & 55 & 3 & None & $\begin{array}{l}\text { 3: Kenab3, Pigs, } \\
\text { Accn1 }\end{array}$ \\
\hline $\begin{array}{l}12860 \\
\text { (nert) }\end{array}$ & $\begin{array}{l}\text { Recessive/ } \\
\text { craniofacial }\end{array}$ & $\begin{array}{l}\text { Spontaneous: } \\
\text { C57BL/6J }\end{array}$ & 121,109 & 105,964 & 30,275 & 1,441 & 639 & 94 & 3 & None & $\begin{array}{l}\text { 4: } \\
\text { 4931406P16Rik, } \\
\text { Shisa7, Nipa1, } \\
\text { Alpk3 }\end{array}$ \\
\hline $\begin{array}{l}13782 \\
(a p h l)\end{array}$ & $\begin{array}{l}\text { Recessive/ } \\
\text { skin, hair }\end{array}$ & $\begin{array}{l}\text { Spontaneous: } \\
\text { MRL/MpJ }\end{array}$ & 182,564 & 156,802 & 57,317 & 554 & 366 & 33 & 1 & None & $\begin{array}{l}\text { 4: Eif2ak3, } \\
\text { Mrpl35, Usp39 } \\
\text { (2) }\end{array}$ \\
\hline $\begin{array}{l}6246 \\
\text { (sunk) }\end{array}$ & $\begin{array}{l}\text { Recessive/ } \\
\text { size }\end{array}$ & $\begin{array}{l}\text { Spontaneous: } \\
\text { A/J }\end{array}$ & 164,053 & 60,051 & 16,508 & 693 & 303 & 25 & 0 & None & None \\
\hline 3485 (frg) & $\begin{array}{l}\text { Recessive/ } \\
\text { craniofacial }\end{array}$ & $\begin{array}{l}\text { Spontaneous: } \\
\text { C57BL/6J, A/J }\end{array}$ & 124,054 & 105,326 & 20,073 & 36 & 22 & 0 & 0 & None & None \\
\hline $\begin{array}{l}4507 \\
(s t n)\end{array}$ & $\begin{array}{l}\text { Recessive/ } \\
\text { craniofacial }\end{array}$ & $\begin{array}{l}\text { Spontaneous: } \\
\text { C57BL/6J }\end{array}$ & 7,523 & 3,079 & 2,338 & 13 & 7 & 0 & 0 & None & None \\
\hline
\end{tabular}

In 6 of the 15 mutant exomes sequenced, candidate mutations in protein coding sequence or splice sites were either not found or could not be validated in additional samples; for three of these, however, candidate mutations in regions annotated at UTRs were identified. ${ }^{a}$ Compared to dbSNP. ${ }^{b}>0.95$ for homozygous samples, $>0.2$ for heterozygous samples. 'compared to unrelated exome data sets. 
sequence data representing the entire candidate region, existing analysis pipelines are not sufficient for discovery of all disease-causative genetic lesions. Moreover, systematic errors in the $\mathrm{mm} 9$ reference sequence or insufficient gene annotation [24] are also likely to contribute to failed mutation discovery, since current analytical approaches rely upon reference and contemporary gene annotation as assumed underlying truth.

In this context, it is notable that the exome-based analysis of human phenotypes that are presumed to be monogenic is also frequently unsuccessful, although such negative results are generally not reported in the literature. Consequently, we anticipate that deeper analysis of the mouse mutants that fail discovery by exome sequencing may also shed light on the nature of both non-coding and cryptic coding mutations that contribute to Mendelian phenotypes in humans.

\section{Conclusions}

Whole exome sequencing is a robust method for mutation discovery in the mouse genome and will be particularly useful for high-throughput genetic analyses of large mutant collections. Due to the nature of the underlying mutations and the current methods available for massively parallel sequence data analysis, ENU mutation discovery via exome sequencing is more successful than spontaneous mutation discovery. In all cases, coarse mapping data (chromosomal linkage) significantly eased validation burden (Table 3); however, fine mapping to chromosomal regions < 10 to $20 \mathrm{Mb}$, while useful, did not provide significant added value (Table 3; Additional file 3). A similar conclusion was drawn by Arnold et al. [5] for mutation discovery via whole genome sequencing. In addition, since the data shown here include mutations on a variety of strain backgrounds, comparison across unrelated exome data sets and to whole genome sequencing data from the Mouse Genomes Project [16] proved critical in reducing the validation burden, especially where mapping data were not available to guide analysis.

Although we are 10 years past the assembly of both the human and mouse genomes, the biological function of the vast majority of mammalian genes remains unknown. We anticipate that the application of exome sequencing to the thousands of immediately available mutant mouse lines exhibiting clinically relevant phenotypes will make a large and highly valuable contribution to filling this knowledge gap.

\section{Materials and Methods}

\section{Exome capture and sequencing}

The following protocol for exome capture and sequencing is the standard protocol generally followed by all sites providing data for proof-of-concept experiments. Site-specific deviations in the standard protocol can be provided upon request. The mouse exome probe pools developed in this study, SeqCap EZ Mouse Exome SR, are commercially available on request from Roche NimbleGen.

\section{DNA extraction}

DNA for high-throughput sequencing was isolated from spleen using a Qiagen DNeasy Blood and Tissue kit (Qiagen, Santa Clarita, CA USA) or by phenol/chloroform extraction of nuclear pellets. Briefly, spleen samples were homogenized in ice-cold Tris lysis buffer $(0.02$ M Tris, pH 7.5, $0.01 \mathrm{M} \mathrm{NaCl}, 3 \mathrm{mM} \mathrm{MgCl}_{2}$ ). Homogenates were then incubated in $1 \%$ sucrose, $1 \%$ NP40 to release nuclei, which were subsequently pelleted by centrifugation at $1,000 \mathrm{rpm}, 4^{\circ} \mathrm{C}$. Isolated nuclei were then extracted by phenol chloroform in the presence of $1 \%$ SDS. DNA for PCR was extracted from small (1 to 2 $\mathrm{mm}$ ) tail biopsies by lysing in $200 \mathrm{ml}$ of $50 \mathrm{mM} \mathrm{NaOH}$ at $95^{\circ} \mathrm{C}$ for 10 minutes. Samples were neutralized by adding $20 \mathrm{ml}$ of $1 \mathrm{M}$ Tris $\mathrm{HCl}, \mathrm{pH} 8.0$ and used directly for PCR amplification.

\section{Capture library preparation and hybridization amplification}

Illumina PE libraries (Illumina, San Diego, CA, USA) were constructed using Illumina's Multiplexing Kit (part number PE-400-1001) with a few modifications. Size selection was done using the Pippin Prep from Sage Science, Inc. (Beverly, MA, USA). The target base pair selection size was set at $430 \mathrm{bp}$. The entire $40 \mu \mathrm{l}$ recovery product was used as template in the pre-hybridization library amplification (using ligation-mediated PCR (LMPCR)). Pre-hybridization LMPCR consisted of one reaction containing $50 \mu \mathrm{l}$ Phusion High Fidelity PCR Master Mix (New England BioLabs, Ipswich, MA, USA; part number F-531L), $0.5 \mu \mathrm{M}$ of Illumina Multiplexing PCR Primer 1.0 (5'-AATGATACGGCGACCACCGAGATCTACACTCTTTCCCTACACGACGCTCTTCCGATCT-3'), $0.001 \mu \mathrm{M}$ of Illumina Multiplexing PCR Primer 2.0 (5'-GTGACTGGAGTTCAGACGTGTGCTCTTCCGATCT-3'), $0.5 \mu \mathrm{M}$ of Illumina PCR Primer, Index 1 (or other index at bases 25-31; 5'CAAGCAGAAGACGGCATACGAGAT(CGTGATG) TGACTGGAGTTC-3'), $40 \mu \mathrm{l}$ DNA, and water up to 100 $\mu \mathrm{l}$. PCR cycling conditions were as follows: $98^{\circ} \mathrm{C}$ for $30 \mathrm{~s}$, followed by 8 cycles of $98^{\circ} \mathrm{C}$ for $10 \mathrm{~s}, 65^{\circ} \mathrm{C}$ for $30 \mathrm{~s}$, and $72^{\circ} \mathrm{C}$ for $30 \mathrm{~s}$. The last step was an extension at $72^{\circ} \mathrm{C}$ for 5 minutes. The reaction was then kept at $4^{\circ} \mathrm{C}$ until further processing. The amplified material was cleaned with a Qiagen Qiaquick PCR Purification Kit (part number 28104) according to the manufacturers instructions, except the DNA were eluted in $50 \mu \mathrm{l}$ of water. DNA was quantified using the NanoDrop-1000 (Wilmington, DE, USA) and the library was evaluated electrophoretically with an Agilent Bioanalyzer 2100 (Santa Clara, CA, USA) using a DNA1000 chip (part number 5067-1504). Sample multiplexing was performed in some cases, after capture and prior to sequencing. 


\section{Liquid phase sequence capture and processing}

Prior to hybridization the following components were added to a $1.5 \mathrm{ml}$ tube: $1.0 \mu \mathrm{g}$ of library material, $1 \mu \mathrm{l}$ of $1,000 \mu \mathrm{M}$ oligo 5' - AATGATACGGCGACCACCGAGATCTACACTCTT TCCCTACACGACGCTCTT CCG ATC*T-3' (asterisk denotes phosphorothioate bond), $1 \mu \mathrm{l}$ of $100 \mu \mathrm{M}$ oligo 5' CAAGCAGAAGACGGCATACGAGATCGTGATGTGACTGGAGTTCAGACGTGTGCT CTTCCGATC*T-3' (bases 25 to 31 correspond to index primer 1), and $5 \mu \mathrm{g}$ of Mouse COT-1 DNA (part number 18440-016; Invitrogen, Inc., Carlsbad, CA, USA). Samples were dried down by puncturing a hole in the $1.5-\mathrm{ml}$ tube cap with a 20 gauge needle and processing in an Eppendorf Vacufuge (San Diego, CA, USA) set to $60^{\circ} \mathrm{C}$ for 20 minutes. To each sample $7.5 \mu \mathrm{l}$ NimbleGen SC Hybridization Buffer (part number 05340721001) and $3.0 \mu \mathrm{l}$ NimbleGen Hybridization component A (part number 05340721001) were added, sample was vortexed for $30 \mathrm{~s}$, centrifuged, and placed in a heating block at $95^{\circ} \mathrm{C}$ for 10 minutes. The samples were again mixed for $10 \mathrm{~s}$, and spun down. This mixture was then transferred to a 0.2-ml PCR tube containing $4.5 \mu \mathrm{l}$ of Mouse Exome Solution Phase probes and mixed by pipetting up and down ten times. The $0.2 \mathrm{ml} \mathrm{PCR}$ tubes were placed in a thermocylcer with heated lid at $47^{\circ} \mathrm{C}$ for 64 to 72 hours. Washing and recovery of captured DNA were performed as described in chapter 6 of the NimbleGen SeqCap EZ Exome SR Protocol version 2.2 (available from the Roche NimbleGen website) [11]. Samples were then quality checked using quantitative PCR as described in chapter 8 of the SR Protocol version 2.2 [10]. Sample enrichment was calculated and used as a means of judging capture success. Mean fold enrichment greater than 50 was considered successful and sequenced. NimbleGen Sequence Capture Control (NSC) quantitative PCR assay NSC0272 was not used to evaluate captures in these experiments.

\section{Post-hybridization LMPCR}

Post-hybridization amplification (for example, LMPCR via Illumina adapters) consisted of two reactions for each sample using the same enzyme concentration as the precapture amplification, but a modified concentration, $2 \mathrm{uM}$, and different versions of the Illumina Multiplexing 1.0 and 2.0 primers were employed: forward primer 5'AATGATACGGCGACCACCGAGA and reverse primer 5'-CAAGCAGAAGACGGCATACGAG. Post-hybridization amplification consisted of 16 cycles of PCR with identical cycling conditions as used in the pre-hybridization LMPCR (above), with the exception of the annealing temperature, which was lowered to $60^{\circ} \mathrm{C}$. After completion of the amplification reaction, the samples were purified using a Qiagen Qiaquick column following the manufacturer's recommended protocol. DNA was quan- tified spectrophotometrically, and electrophoretically evaluated with an Agilent Bioanalyzer 2100 using a DNA1000 chip (Agilent). The resulting post-capture enriched sequencing libraries were diluted to $10 \mathrm{nM}$ and used in cluster formation on an Illumina cBot and PE sequencing was done using Illumina's Genome Analyzer IIx or Illumina HiSeq. Both cluster formation and PE sequencing were performed using the Illumina-provided protocols.

\section{High-throughput sequencing data analysis Mapping, SNP calling and annotation}

The sequencing data were mapped using Maq, BWA (Burrows-Wheeler alignment tool) and/or GASSST (global alignment short sequence search tool) and SNP calling was performed using SAMtools [25] and/or GenomeQuest [26]. SNP annotation was performed using GenomeQuest, custom scripts and Galaxy tools. Alignments were visualized with the UCSC genome browser, Integrated Genomics Viewer (Broad Institute) and/or SignalMap (Roche NimbleGen).

\section{Validation}

Candidate mutations were validated by PCR amplification and sequencing of affected and unaffected samples if available from the mutant colony or from archived samples. Sequencing data were analyzed using Sequencher 4.9 (Gene Codes Corp., Ann Arbor, MI, USA). Primers were designed using Primer3 software [27].

$R T-P C R$

Total RNA was isolated from heterozygous and homozygous tail biopsies and/or embryos using the RNeasy Mini Kit (Qiagen) according to the manufacturer's protocols. Total RNA $(1 \mu \mathrm{g})$ was reverse transcribed into cDNA using the SuperScript III First-Strand Synthesis SuperMix for quantitative RT-PCR (Invitrogen) according to the manufacturer's protocols. cDNA $(3 \mu \mathrm{l})$ was used as template in a $30 \mu \mathrm{l} \mathrm{PCR}$ with the following cycling conditions for all primers $(0.4 \mu \mathrm{M}$ final concentration): $94^{\circ} \mathrm{C}(45 \mathrm{~s}), 56^{\circ} \mathrm{C}(45 \mathrm{~s}), 72^{\circ} \mathrm{C}(45 \mathrm{~s})$ for 30 cycles. Primers used for Cleft were Cleft_11-14f ( $5^{\prime}$ CTGGAAAACCTGGTGACGAC) and Cleft_11-14R (5'ACCAGCTTCCCCCTTAGC).

\section{Additional material}

Additional file 1: Summary statistics for the alpha and beta exome probe pools.

Additional file 2: Comparison of $2 \times 76$-bp datasets from four independent captures of female $\mathrm{C} 56 \mathrm{BL} / 6 \mathrm{~J}$ DNA and one capture of male C57BL/6J compared to alpha data from one capture of male C57BL/6J.

Additional file 3: Additional data on mutant exomes sequenced in this study. Genetic background, size of mapped intervals, genotype of sequenced sample and percentage of SNVs identified are provided. 
Additional file 4: Data generated from exome sequencing of mutant and control exomes $(2 \times 40 \mathrm{bp}, 2 \times 76$ Illumina or $2 \times 100$ HiSeq).

\section{Additional file 5: Seventeen variants passing filter in a C57BL/6J} exome. The genome coordinate and gene annotation for each variant are provided. Comparison of these variants with the high-throughput sequencing data for 17 inbred strains available from Sanger Mouse Genomes Project revealed three exonic SNVs that are likely unique to the C57BL/6J exome.

\section{Abbreviations}

bp: base pair; dbSNP: Single Nucleotide Polymorphism Database; ENU: Nethyl-N-nitrosourea; INDEL: insertions/deletion; LMPCR: ligation-mediated PCR; NCBI: National Center for Biotechnology Information; PCR: polymerase chain reaction; PE: paired-end; RefSeq: NCBI Reference Sequence; RT-PCR: reverse transcriptase polymerase chain reaction; SNV: single nucleotide variant; UTR: untranslated region; VEGA: The Vertebrate Genome Annotation database.

\section{Acknowledgements}

We are grateful to the Mouse Genome Informatics team at The Jackson Laboratory for providing custom queries of the Mouse Genome Database. We would also like to thank Belinda Harris, Son Yong Karst, Louise Dionne, Pat Ward-Bailey and Coleen Kane of the The Jackson Laboratory Mutant Mouse Resource for animal husbandry and technical assistance. We also thank Lindsay Felker, Alexandra MacKenzie, and Choli Lee at the University of Washington for analytical and technical assistance. We are grateful to the Illumina High Throughput Sequencing Service and the DNA Resource at The Jackson Laboratory for providing sequencing support and archived DNA samples. The repro7 mutant was obtained from The Reproductive Genomics program at The Jackson Laboratory (NICHD P01 HD42137) and was sequenced at the Broad Institute under the Mouse Mutant Re-sequencing Project [28]. This work was supported in part by the Australian Phenomics Network. This work was also supported in part by The Mouse Mutant Resource and the Craniofacial Resource at The Jackson Laboratory, NIH-NCRR RR001183, NEI EY015073. MSS was supported by a generous contribution from The Don Monti Memorial Research Foundation. SWL is a Howard Hughes Medical Institute Investigator and is also supported in part by the Mouse Models of Human Cancer Consortium, grant 5 U01 CA105388.

\section{Author details}

${ }^{1}$ The Jackson Laboratory, 600 Main St, Bar Harbor, ME 04609, USA. ${ }^{2}$ Baylor College of Medicine, Department of Molecular and Human Genetics, One Baylor Plaza R804, Houston, Texas 77030, USA. ${ }^{3}$ Cold Spring Harbor Laboratory, One Bungtown Road, Cold Spring Harbor, NY 11724, USA. ${ }^{4}$ Roche NimbleGen, Inc. Madison, WI 53719, USA. ${ }^{5}$ National Center for Genome Analysis (CNAG), Parc Científic de Barcelona, Torre I, Baldiri Reixac, 408028 Barcelona, Spain. ${ }^{6}$ Walter and Eliza Hall Institute, 1G Royal Parade, Parkville, Victoria 3052, Australia. ${ }^{7}$ University of Washington, Department of Pediatrics, Division of Craniofacial Medicine and Seattle Children's Craniofacial Center, 4800 Sand Point Way NE, Seattle, WA 98105, USA. ${ }^{8}$ Regeneron Pharmaceuticals Inc., 777 Old Saw Mill River Road, Tarrytown, NY

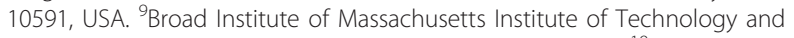
Harvard, 5 Cambridge Center, Cambridge, MA 02142, USA. ${ }^{10}$ University of Washington, Department of Genome Sciences, Foege Building S-250, Box 355065, 3720 15th Ave NE, Seattle, WA 98195-5065, USA.

\section{Authors' contributions}

$J$ J, LGR, JS, BTK, IG, JH, and SWL participated in the conception of the mouse exome design. $C B$ and JR created and provided the gene list that was the basis for the exome design. JS, SS, EM, FDP, and KLT provided sequencing support. MSS, LGR, SAM, LRD, DEB, MLC, TCC, and SWL provided mutant samples. WH, CH, DG, HF, GG, MB, LR, RRC, FJP, and MC performed sample preparation, exome capture, PCR and RT-PCR validation. YD, MD, DG, and TR provided sequence analysis and bioinformatics support. LGR, JS and JJ conceived of the study, and participated in its design and coordination and drafted the manuscript. All authors read and approved the final manuscript.

\section{Competing interests}

The authors from Roche NimbleGen recognize a competing interest in this publication as employees of the company. The other authors declare that they have no competing interests.

Received: 27 May 2011 Revised: 4 August 2011

Accepted: 14 September 2011 Published: 14 September 2011

\section{References}

1. Mouse Genome Informatics.. [http://www.informatics.jax.org/mgihome/ homepages/stats/all_stats.shtml].

2. Shendure J, Ji H: Next-generation DNA sequencing. Nat Biotechnol 2008, 26:1135-1145

3. Zhang Z, Alpert D, Francis R, Chatterjee B, Yu Q, Tansey T, Sabol SL, Cui C, Bai Y, Koriabine M, Yoshinaga Y, Cheng JF, Chen F, Martin J, Schackwitz W, Gunn TM, Kramer KL, De Jong PJ, Pennacchio LA, Lo CW: Massively parallel sequencing identifies the gene Megf8 with ENU-induced mutation causing heterotaxy. Proc Natl Acad Sci USA 2009, 106:3219-3224.

4. D'Ascenzo M, Meacham C, Kitzman J, Middle C, Knight J, Winer R, Kukricar M, Richmond T, Albert TJ, Czechanski A, Donahue LR, Affourtit J, Jeddeloh JA, Reinholdt L: Mutation discovery in the mouse using genetically guided array capture and resequencing. Mamm Genome 2009, 20:424-436.

5. Arnold CN, Xia Y, Lin P, Ross C, Schwander M, Smart NG, Muller U, Beutler B: Rapid identification of a disease allele in mouse through whole genome sequencing and bulk segregation analysis. Genetics 2011, 187:633-641.

6. Ng SB, Bigham AW, Buckingham KJ, Hannibal MC, McMillin MJ, Gildersleeve HI, Beck AE, Tabor HK, Cooper GM, Mefford HC, Lee C, Turner EH, Smith JD, Rieder MJ, Yoshiura K, Matsumoto N, Ohta T, Niikawa N, Nickerson DA, Bamshad MJ, Shendure J: Exome sequencing identifies MLL2 mutations as a cause of Kabuki syndrome. Nat Genet 2010, 42:790-793.

7. Ng SB, Buckingham KJ, Lee C, Bigham AW, Tabor HK, Dent KM, Huff CD, Shannon PT, Jabs EW, Nickerson DA, Shendure J, Bamshad MJ: Exome sequencing identifies the cause of a mendelian disorder. Nat Genet 2010, 42:30-35.

8. Zuchner S, Dallman J, Wen R, Beecham G, Naj A, Farooq A, Kohli MA, Whitehead PL, Hulme W, Konidari I, Edwards YJ, Cai G, Peter I, Seo D, Buxbaum JD, Haines JL, Blanton S, Young J, Alfonso E, Vance JM, Lam BL, Pericak-Vance MA: Whole-exome sequencing links a variant in DHDDS to retinitis pigmentosa. Am J Hum Genet 2011, 88:201-206.

9. Ostergaard P, Simpson MA, Brice G, Mansour S, Connell FC, Onoufriadis A, Child AH, Hwang J, Kalidas K, Mortimer PS, Trembath R, Jeffery S: Rapid identification of mutations in GJC2 in primary lymphoedema using whole exome sequencing combined with linkage analysis with delineation of the phenotype. J Med Genet 2011, 48:251-255.

10. Walsh T, Shahin H, Elkan-Miller T, Lee MK, Thornton AM, Roeb W, Abu Rayyan A, Loulus S, Avraham KB, King MC, Kanaan M: Whole exome sequencing and homozygosity mapping identify mutation in the cell polarity protein GPSM2 as the cause of nonsyndromic hearing loss DFNB82. Am J Hum Genet 2010, 87:90-94.

11. Bainbridge $M N$, Wang $M$, Burgess $D L$, Kovar C, Rodesch MJ, D'Ascenzo M, Kitzman J, Wu YQ, Newsham I, Richmond TA, Jeddeloh JA, Muzny D, Albert TJ, Gibbs RA: Whole exome capture in solution with $3 \mathrm{Gbp}$ of data. Genome Biol 2010, 11:R62.

12. Choi M, Scholl UI, Ji W, Liu T, Tikhonova IR, Zumbo P, Nayir A, Bakkaloglu A, Ozen S, Sanjad S, Nelson-Williams C, Farhi A, Mane S, Lifton RP: Genetic diagnosis by whole exome capture and massively parallel DNA sequencing. Proc Natl Acad Sci USA 2009, 106:19096-19101.

13. Blake JA, Bult CJ, Kadin JA, Richardson JE, Eppig JT: The Mouse Genome Database (MGD): premier model organism resource for mammalian genomics and genetics. Nucleic Acids Res 2011, 39:D842-848.

14. Mouse Exome Gene List.. [ftp://ftp.jax.org/ Genome_Biology_mouse_exomes/mouse_exome_genes.xls.zip].

15. Mouse Exome Design.. [ftp://ftp.jax.org/Genome_Biology_mouse_exomes/ 100803_MM9_exome_rebal_2_EZ_HX1.gff.bz2].

16. Mouse Genomes Project.. [http://www.sanger.ac.uk/resources/mouse/ genomes/]. 
17. Brancho D, Ventura JJ, Jaeschke A, Doran B, Flavell RA, Davis RJ: Role of MLK3 in the regulation of mitogen-activated protein kinase signaling cascades. Mol Cell Biol 2005, 25:3670-3681.

18. Hitotsumachi S, Carpenter DA, Russell WL: Dose-repetition increases the mutagenic effectiveness of $\mathrm{N}$-ethyl- $\mathrm{N}$-nitrosourea in mouse spermatogonia. Proc Natl Acad Sci USA 1985, 82:6619-6621.

19. Leung AW, Wong SY, Chan D, Tam PP, Cheah KS: Loss of procollagen IIA from the anterior mesendoderm disrupts the development of mouse embryonic forebrain. Dev Dyn 2010, 239:2319-2329.

20. Donahue LR, Chang B, Mohan S, Miyakoshi N, Wergedal JE, Baylink DJ, Hawes NL, Rosen CJ, Ward-Bailey P, Zheng QY, Bronson RT, Johnson KR, Davisson MT: A missense mutation in the mouse Col2a1 gene causes spondyloepiphyseal dysplasia congenita, hearing loss, and retinoschisis. J Bone Miner Res 2003, 18:1612-1621.

21. Kile BT, Hentges KE, Clark AT, Nakamura H, Salinger AP, Liu B, Box N, Stockton DW, Johnson RL, Behringer RR, Bradley A, Justice MJ: Functional genetic analysis of mouse chromosome 11. Nature 2003, 425:81-86.

22. Zheng B, Sage M, Cai WW, Thompson DM, Tavsanli BC, Cheah YC, Bradley A: Engineering a mouse balancer chromosome. Nat Genet 1999, 22:375-378.

23. Hentges KE, Nakamura $H$, Furuta Y, Yu Y, Thompson DM, O'Brien W, Bradley A, Justice MJ: Novel lethal mouse mutants produced in balancer chromosome screens. Gene Expr Patterns 2006, 6:653-665.

24. Boles MK, Wilkinson BM, Wilming LG, Liu B, Probst FJ, Harrow J, Grafham D, Hentges KE, Woodward LP, Maxwell A, Mitchell K, Risley MD, Johnson R, Hirschi K, Lupski JR, Funato Y, Miki H, Marin-Garcia P, Matthews L, Coffey AJ, Parker A, Hubbard TJ, Rogers J, Bradley A, Adams DJ, Justice MJ: Discovery of candidate disease genes in ENU-induced mouse mutants by largescale sequencing, including a splice-site mutation in nucleoredoxin. PLOS Genet 2009, 5:e1000759.

25. Galaxy.. [http://main.g2.bx.psu.edu]

26. GenomeQuest.. [http://www.genomequest.com/].

27. Primer3.. [http://frodo.wi.mit.edu/primer3/].

28. Mouse Mutant Re-sequencing Project.. [http://www.broadinstitute.org/ scientific-community/science/projects/mammals-models/mouse/mousemutant-resequencing].

doi:10.1186/gb-2011-12-9-r86

Cite this article as: Fairfield et al: Mutation discovery in mice by whole exome sequencing. Genome Biology 2011 12:R86.

\section{Submit your next manuscript to BioMed Central and take full advantage of:}

- Convenient online submission

- Thorough peer review

- No space constraints or color figure charges

- Immediate publication on acceptance

- Inclusion in PubMed, CAS, Scopus and Google Scholar

- Research which is freely available for redistribution

Submit your manuscript at www.biomedcentral.com/submit
Biomed Central 